\title{
La autoevaluación en la educación a distancia
}

\author{
Luis Gerardo Argoty Hidalgo ${ }^{1}$ \\ Fedra Lorena Ortiz Benavides ${ }^{2}$ \\ Clemencia Alava Viteri ${ }^{3}$
}

\begin{abstract}
Resumen
En este artículo se presenta un análisis documental, en el cual se revisan ideas de teóricos importantes en el ámbito de la educación a distancia, sobre evaluación formativa, especialmente lo referido a autoevaluación bajo concepciones constructivistas. Además, desde el punto de vista de la tecnología instruccional, se examinarán ideas sobre la autoevaluación dentro del diseño instruccional y las estrategias y acciones propuestas para que los estudiantes la empleen para mejorar su aprendizaje.
\end{abstract}

Palabras claves: evaluación formativa, autoevaluación, autorregulación, meta-cognición. 


\title{
Self-evaluation in distance education
}

\begin{abstract}
This article presents a review on the fundamental theoris in the field of distance learning, on the subject of formative assessment, especially concerning constructivism-based self-evaluation. Additionally from an Instructional Technology approach, ideas on self-evaluation within instructional design will be examined, as well as the strategies and actions proposed, so that students may use them to improve learning.
\end{abstract}

Key Words: formative assessment, self-evaluation, self-regulation, metacognition.

Recibido: 11 de agosto 2011

Aceptado: 24 de noviembre 2011

\section{lntroducción}

Este artículo se enmarca dentro del campo de la Educación a Distancia y la Tecnología Instruccional. Desde la Educación a Distancia se incursionará en la apreciación que tienen los teóricos, docentes y estudiantes sobre la evaluación formativa, la cual se define desde las concepciones constructivistas como el proceso que el sistema educativo debe proporcionar de manera integral (aspectos motrices, equilibrio personal y emocional, inserción social y relaciones interpersonales) a los estudiantes (Ahumada Acevedo, 2001). . Esto implica conferir un carácter activo al aprendizaje para que éste sea consecuencia de una construcción personal donde intervienen otros agentes culturales.

Por otra parte, desde la Tecnología Instruccional el diseño de cursos se ha respaldado en modelos que las diversas instituciones de educación a distancia han impulsado para los procesos formativos de su oferta educativa. En esa medida, el centro de atención ha sido el diseño instruccional de las actividades relacionadas con objetivos de aprendizaje y con recursos informativos para su desarrollo. Aun cuando en la mayoría de los cursos se destaca la importancia de la evaluación del aprendizaje y se pone especial cuidado en su diseño, poco se ha efectuado para que los estudiantes aprendan a autoevaluar sus producciones y para que reconozcan su proceso formativo.

Por lo anterior, es importante analizar los principales aspectos que se deben tener en cuenta para la conformación de la evaluación del aprendizaje, de tal forma 
que la comunidad educativa pueda valorar la calidad de lo elaborado por sus estudiantes y les ofrezca a su vez la posibilidad de identificar cómo se les evaluará. Particularmente, con el fin de avanzar en el proceso de aprender a autoevaluarse, lo que contribuirá de manera significativa en su formación.

Esta reseña bibliográfica abarca el estudio de documentos que de manera general abordan los conceptos de «evaluación educativa», «evaluación pedagógica», «evaluación formativa» y específicamente, «autoevaluación», así como el estudio de algún software que apoye estas prácticas. Igualmente se analizan los mismos elementos desde el punto de vista de la Educación a Distancia. La conceptualización que sobre la autoevaluación se tenga y si esto conlleva de manera significativa a determinar elementos que se utilicen en el campo de la Tecnología Instruccional con el fin de procurar que el docente y el estudiante de esta modalidad, se forman de manera efectiva tanto en la enseñanza como en el aprendizaje.

\section{Metodología}

El material estudiado para realizar esta reseña bibliográfica se buscó en función al tema a tratar. En la búsqueda se tuvieron en cuenta las palabras clave «autoevaluación formativa», «evaluación formativa», «evaluación educativa», «evaluación pedagógica» (en lo posible dentro de la modalidad de educación a distancia). Se trata de hacer un diagnóstico de manera general sobre la manera en que se trabaja el proceso de autoevaluación desde su realización por parte de los estudiantes o desde el punto de vista de mediación cuando es utilizada por los diseñadores docentes para lograr que el estudiante la utilice como parte de su formación. Este proceso se aborda entonces, como algo inherente que debe estar presente en cada momento en el proceso de construcción de cada estudiante para que éste determine con toda responsabilidad y criterio los aspectos en los cuales existen debilidades y aquellos aspectos que considera fortalezas con el fin de tomar los correctivos del caso.

Se revisaron las bases de datos de la N.S.U, revistas electrónicas especializadas, internet por intermedio de los buscadores Google, Bing desde el buscador normal hasta utilizando los buscadores especializados en relación a lo académico.

También se extendió la búsqueda a libros de la editorial Magisterio: Interrogar o examinar de Juvenal Nieves Herrera, Evaluación escolar ¿Resultados o procesos? (1996) de Mauricio Pérez Abril y Guillermo Bustamante Zamudio, Evaluación Integral por procesos, una experiencia construida en el aula (1996) de Cayetano Estévez Solano y La evaluación como experiencia total de Hugo Cerda Gutierrez. Así mismo se revisó el documento Estructura Académica de la educación a Distancia de la Fundación Universitaria San Martín, Universidad que trabaja la Educación a Distancia semipresencial. 
El procedimiento para el análisis fue el siguiente:

- Anotar los datos bibliográficos.

- Determinar mediante la revisión del índice cuáles son las partes, capítulos, incisos y subincisos del libro, revista electrónica, pagina web, etc.

- Leer la introducción, prólogo y conclusiones.

- Leer cada capítulo para enterarse de su contenido.

- Hacer, en caso necesario, algunas citas textuales breves.

Los criterios de evaluación se encaminan a escoger aquellos documentos que más aporten sobre el tema específico de autoevaluación del aprendizaje en la modalidad de educación a distancia; en segundo lugar aquellos documentos que aporten a la evaluación educativa, evaluación pedagógica y educación en la modalidad a distancia y en último lugar, aquellas que hacen referencia a la evaluación en general.

\section{Resultados}

La evaluación es una acción que es inherente al ser humano ya que continuamente se dan opiniones, se emiten juicios, se exponen conceptos que son evaluaciones y ayudan a mejorar los aspectos que se presentan en la cotidianidad. Por lo tanto, la evaluación es algo interno que responde a las necesidades del ser humano frente al desarrollo cognoscitivo, socio-afectivo y psicomotriz.

Desde el punto de vista del campo educativo, varios de los autores consultados coinciden en considerar la evaluación como un proceso complejo. Estos autores expresan su punto de vista de la evaluación ya sea desde la enseñanza y/o desde el aprendizaje, lo que conlleva a pensar que la visión de la evaluación se complica aún más dependiendo de la concepción que se tenga de ella.

Barberà (2008) considera completa una evaluación si abarca las siguientes dimensiones: evaluación del aprendizaje, evaluación para el aprendizaje, evaluación como aprendizaje y evaluación desde el aprendizaje. Esta autora considera importante rescatar para la modalidad a distancia de la educación esta concepción completa de evaluación y destaca la importancia de que se adhiera a las prácticas evaluativas propias de la Educación a Distancia tales como la evaluación automática, la evaluación enciclopédica y la evaluación colaborativa. García Aretio (1998) hace un estudio sobre los indicadores para la evaluación de la enseñanza en una universidad a distancia y da a entender la evaluación como 
una actividad compleja y amplia, realizada durante todo el proceso formativo profesional. Por su parte. García Ramos (1986) y Mateo (2000) entienden la evaluación como aquel conjunto de procesos sistemáticos de recogida, análisis e interpretación de información válida y fiable que en comparación con una referencia o criterio, nos permita llegar a una decisión que favorezca la mejora del objeto evaluado. Rodríguez (2005) trata la evaluación educativa desde el punto de vista formativo y sumativocitando varias experiencias y herramientas informáticas al uso en el contexto universitario internacional. Aboga por la exigencia de una formación de calidad constituida por una eficaz evaluación ya que ésta permite determinar, entre otros aspectos, el nivel de conocimientos y habilidades que haya adquirido el estudiante.

Ryan, Scott, Freeman y Patel plantean la evaluación de los aprendizajes como «un proceso mediante el cual los estudiantes ganan una comprensión de sus propias competencias y progreso así como un proceso mediante el cual son calificados» (2002: ). Para ellos la evaluación formativa tiene como propósito «obtener información acerca del progreso de un estudiante en particular, para darle retroalimentación a los estudiante y a sus profesores» (Ryan et al., 2002: ).

Morgan y O'Reilly conciben la evaluación como «la maquinaria que dirige y da forma al aprendizaje, más que simplemente un evento final que califica y reporta el desempeño» (2002: ). Consideran que este enfoque permite encontrar nuevas oportunidades para promover, a través de la evaluación, tipos de aprendizaje más útiles y deseables. La «Evaluación formativa comprende todas aquellas actividades diseñadas para motivar, para aumentar la comprensión y para proporcionar a los estudiantes una indicación de sus progresos» (Morgan y O’Reilly, 2002: ).

Por su parte Cayetano Estévez (1996) hace una referencia general a lo que se entiende por evaluación integral por procesos. Señala que al estudiar la evaluación a lo largo de la historia, ésta ya no se percibe como algo interno sino como algo externo que habitualmente genera temor en los estudiantes. Anteriormente sólo se consideraba como medición de conocimientos a través de pruebas estandarizadas que brindaban resultados de tipo cuantitativo; posteriormente se dio una evaluación en la que era necesario establecer objetivos; finalmente se empezó a reflexionar sobre el modo en que se hace la evaluación, trayendo consigo formas alternativas de evaluación, donde el estudiante no sólo está constituido por conocimientos sino que se le considera una persona que tiene experiencia previas, un ser social que no se limita únicamente a repetir los conceptos dados por el profesor.

Generalmente la evaluación de los aprendizajes hace referencia al logro de objetivos propuestos para la enseñanza. En la Educación a Distancia es importante tener presente que el estudiante no tiene contacto directo con el tutor, por lo que en cada encuentro debe haber asesoría y retroalimentación, es decir, acompañamiento desde el punto de vista formativo; así es que dentro del sistema se presentan varios tipos evaluación que suministran información acerca del avance del estudiante. 
Hay que destacar que la evaluación por procesos es la adecuada en estos casos, ya que permite que se realice de manera permanente en el transcurso de la formación del estudiante ayudando a orientar su aprendizaje.

Señala Cayetano Estévez (1996) que los procesos a evaluar en educación se encaminan al mejoramiento y el crecimiento de los sujetos que están formándose. Estos procesos corresponden al desempeño, al desarrollo de aptitudes y el rendimiento, acompañados además de las dimensiones prácticas, valorativas y teóricas. También menciona que la evaluación por procesos busca una valoración cualitativa permanente, sistemática, analizando la práctica educativa del estudiante en su totalidad y en la dinámica misma de su proceso.

En la Educación a Distancia hay muchas opciones para evaluar la actividad educativa de los estudiantes. Estas pueden ser orales (como entrevistas o socializaciones en las que el estudiante discute acerca de los temas tratados) o escritas (cuestionarios, ejercicios, ensayos, informes). Según quien la realiza pueden ser heteroevaluación (cuando la realiza la persona experta en forma presencial o a través de un sistema) o autoevaluación (la acción que realiza el mismo estudiante y que tiene como propósito mejorar su propio proceso de formación).

Con respecto a lo descrito anteriormente por los diferentes autores consultados, se puede dejar en claro que la evaluación es un proceso muy complejo y que en la comprensión de este proceso intervienen factores de tipo psicológico, epistemológico, etc., así como diversas concepciones de enseñanza y/o aprendizaje. Por lo tanto, el estudio de la evaluación del aprendizaje resulta relevante para delimitar su función como un campo abierto de exploración dentro del campo de la Educación a Distancia y para que se siga estudiando desde lo educativo, didáctico y pedagógico hasta el interior de la Tecnología Educativa, donde la persona que diseñe espacios de aprendizaje a través de cualquier medio de comunicación y empleando los diversos recursos o tipos de pruebas de los que dispone el tutor estén acordes a la finalidad de evaluación de aprendizaje que se tiene en este momento. Se debe dejar claro que quien diseña y programa actividades de este tipo es un pedagogo conocedor de los procesos de evaluación y si no lo es, debe apoyarse o trabajar con una persona que tenga claro en qué consiste la evaluación desde lo didáctico. Así aportará a la formación del estudiante y permitirá que la evaluación del aprendizaje se convierta efectivamente en una oportunidad para construir más, para detectar debilidades y fortalezas y para investigar permanentemente los instrumentos, medios y concepciones que vayan surgiendo en la práctica.

Por otra parte, con respecto al concepto específico de autoevaluación, el concepto que se quiere estudiar para determinar la claridad de su manejo, es importante señalar las posibilidades que brinda y las tendencias que existen de acuerdo a las concepciones de aprendizaje que se tienen actualmente. Al respecto Gustavo 
Mantilla Ortiz (2009) escribe que la autoevaluación debe ser pensada como un proceso mediante el cual el propio estudiante reconoce sus logros y limitaciones en el aprendizaje. Además indica que su uso adecuado en el módulo establece una motivación y un apoyo al estudiante; además, lo inicia en el desarrollo de actividades preparatorias para enfrentar posteriormente las pruebas formativas de evaluaciones parciales y finales del módulo. Los requisitos básicos para la preparación de una buena autoevaluación es que ésta sea capaz de mejorar y reorientar los procesos de aprendizaje. Para ello debe haber plena coherencia y armonía entre los objetivos planeados, la actividad docente desarrollada y el contenido de la evaluación, lo que lleva a preparar un conjunto de actuaciones técnicas en la construcción de las pruebas a utilizar. Debe haber además continuidad en la recolección de información capaz de favorecer un proceso de aprendizaje, en paralelo con la enseñanza. El uso de esa información y no control y sanción de los resultados favorece la reorientación de los procesos de aprendizaje.

Rocío Quesada (2006) destaca también la importancia de la evaluación formativa, especialmente en experiencias de autoevaluación, es decir, cuando es el propio sujeto que aprende quien realiza su propia evaluación. Como muy bien señalan Cabero y Gisbert, se debe pensar la autoevaluación como «un conjunto de actividades autocorrectivas o acompañadas de soluciones que permiten al estudiante comprobar el tipo y grado de aprendizaje respecto de cada uno de los objetivos de la acción formativa» (2002: ).

Entre las razones más destacadas para la buena práctica de la autoevaluación son la autonomía que se le concede al educando, mayor protagonismo en su aprendizaje, mayor responsabilidad, un fomento en el estudiante de la capacidad de valorar su proceso educativo y el desarrollo de una actividad crítica importante -particularmente si se aprovechan las ventajas de las TICs al facilitar una retroalimentación inmediata.

La autoevaluación, predomina en la educación a distancia, entre sus bondades está el hecho de que aumenta la responsabilidad, la implicación del discente en la evaluación e incide en sus procesos cognitivos. La idea de la autoevaluación es que los estudiantes reflexionen sobre su aprendizaje y que articulen las consecuencias de cada reflexión. Estas actividades de autoevaluación pueden ser utilizadas por el profesor. Los tipos de tareas que se dejen para la autoevaluación deben dar la oportunidad al discente de valorar inmediatamente si las respuestas son correctas o no y que errores cometió. Por lo que se sugieren tareas de respuestas cerradas.

Los anteriores conceptos de autoevaluación dejan claro que ésta es un medio que permite el mejoramiento de lo que se está aprendiendo. El propio sujeto que aprende es capaz de reflexionar y cuestionarse sobre lo que ha aprendido y puede determinar los respectivos correctivos en caso de que se determinen o encuentren debilidades. También es importante destacar que es un recurso que puede ayudar al docente, profesor o tutor a determinar qué aspectos se deben 
enfatizar, profundizar o trabajar nuevamente, siempre y cuando se tenga acceso a esta información. Desde el punto de vista de la Tecnología Educativa resulta relevante cerciorarse que los elementos que se utilizan en el diseño y práctica de la evaluación beneficien al estudiante y al educador por igual. Al estudiante, para que esté en capacidad de determinar los avances que hay en el proceso de formación, y al educador para que pueda determinar el tipo de apoyo que el estudiante necesita. Queda pendiente que se mencionen de manera específica situaciones que puedan apoyar de manera clara y específica a su aplicación en la modalidad de Educación a Distancia. Se observa que hay una buena conceptualización sobre los fines que se persiguen pero es necesarioobtener resultados más tangibles, más aplicables para que sean utilizados como apoyo al aprendizaje.

En la revisión bibliográfica se encontraron además, experiencias en las cuales se emplea algún software utilizado para la práctica de la autoevaluación en el proceso de aprendizaje. María José Rodríguez (2005), aborda el uso de la informática en el proceso de evaluación de aprendizajes e indica que al realiza una búsqueda de estos términos en las bases de datos y revistas especializadas, se encuentran una variedad de expresiones que conllevan matices diferenciales para conceptos afines (Stephens, 2001): Computer Assisted Assessment (CAA), Computer Based Assessment (CBA), Computer Aided Instruction (CAI), Computer Based Testing (CBT), Computer Assissted Testing (CAT), etc.

La evaluación del rendimiento de los estudiantes en los sistemas basados en teleformación es uno de los temas claves como en cualquier programa de formación. Sin embargo, la mayor parte de los esfuerzos que se realizan sobre evaluación en línea del aprendizaje se centran en desarrollar herramientas informáticas como Jquiz, Test Creator, Web Work-Shett, WebTest, Question Mark Perception, etc., donde los planteamientos sobre evaluación están totalmente desligados del diseño general del programa de formación, salvo en algunos casos específicos ligados a sistemas de autor. En este sentido podemos afirmar que estas herramientas de evaluación están constituidas por software diseñados para la creación de pruebas tipo test de respuesta cerrada, desarrolladas generalmente desde planteamientos tecnológicos ysin bases pedagógicas. A esta conclusión también llegan García et al. (2002) cuando señalan que se han desarrollado entornos centrados de manera casi exclusivamente en la presentación de contenidos. No tienen en cuenta todo el proceso enseñanza-aprendizaje y, en caso de incluir un módulo de evaluación, éste prácticamente se limita a los test como único instrumento de evaluación. Además, carecen de base pedagógica y no se adaptan al estudiante. En ese escrito la autora indica que de acuerdo a las nuevas corrientes constructivista se debe evaluar la participación y contrastar si los alumnos han alcanzado determinados aprendizajes y por tanto si se han alcanzado los objetivos del curso. De igual forma se plantea que el estudiante reciba retroalimentación de inmediato para observar cómo está aprovechando el curso y se propone la autoevaluación como elemento motivador. 
Hay que tener en cuenta que en la enseñanza en línea, se dispone de muchos materiales para realizar la evaluación de los estudiantes, ya que gran parte de la comunicación se realiza por escrito.

Como señalan García Carrasco et al. (2002), la evaluación es una parte indispensable al interior del proceso enseñanza-aprendizaje, como medida de la consecución de los objetivos de aprendizaje por parte del estudiante y también como control de la calidad del proceso. Sin embargo, los instrumentos de evaluación actuales presentan muchas limitaciones en el contexto lectoescritor que pueden ser superadas, en parte, por las nuevas tecnologías de la información y la comunicación. Además están surgiendo nuevas tecnologías que permiten la construcción de modelos más completos que se aproximan más a los criterios de evaluación. La Web es un espacio universal de información, pero se trata de convertirlo en un espacio universal de conocimiento.

Por otra parte, con respecto a experiencias de autoevaluación y uso de las TICs se pueden mencionar dos desarrolladas en la Facultad de Educación de la Universidad de Salamanca, con estudiantes que han cursado la asignatura de Investigación Evaluativa en la Licenciatura de Pedagogía en el año 2006. En una de las experiencias se empleó un software específico de evaluación a través de Internet: Perception de Question Mark, y en la otra se utilizó el gestor de evaluación de unaplataforma de acceso libre: Moodle. Se utilizaron entonces, dos sistemas cuya diferencia reside en que el primero fue creado para tareas especialmente evaluativas, mientras que en elsegundo el objetivo principal no es la evaluación (aunque contiene un módulo que permite gestionar actividades propias de este campo). Las experiencias de evaluación online que se desarrollaron con Perception y con Moodle se basaron en el ejercicio de autoevaluaciones puesto que se consideró que son muchas las razones que justifican la incorporación de la autoevaluación como instrumento y medio de ayuda a los estudiantes en sus aprendizajes. El objetivo formulado fue demostrar la eficacia de las TICs en la evaluación de los alumnos universitarios. Los estudiantes que participaron en la prueba confirmaron que aprendieron mejor y que los instrumentos de evaluación les facilitó la comprensión de los conceptos de la materia a la vez que les ayudo a responsabilizarse de su propio aprendizaje. Estas autoevaluaciones informatizadas por tanto, fueron un importante instrumento de apoyo al estudio y aprendizaje de los alumnos universitarios. Con ambas experiencias se pretendió ayudar a los estudiantes a entender la materia y mejorar sus hábitos de estudio, haciéndolos responsables de sí mismos y logrando que sean autónomos en su proceso de aprendizaje.

Del mismo modo se resaltó la importancia de incorporar las TICs ysus potencialidades en el proceso educativo. Por tanto la conclusión final es que este tipo de pruebas de autoevaluación a través de Internet, utilizando tanto Perception como Moodle, no son para valorar el rendimiento de los alumnos, sino para crear una herramienta apoyo al estudio (Olmos , 2006). 
También se encontró otro documento de Díaz et al. (s.f.) quienes provenientes de la Universidad de Oviedo, España, realizaron una experiencia con una herramienta E-valúate, que hace posible una evaluación individualizada, continua y adaptativa en la que cada estudiante consigue información elaborada de sus errores con respecto a objetivos, contenido de la materia y causas del error. Asimismo, E-valúate registra y compara resultados a nivel de grupo de estudiantes, lo que permite obtener conclusiones acerca del grado de cumplimiento de objetivos alcanzados en la materia. Por lo tanto, proporciona información al profesor acerca de la calidad de la transferencia de información, sacando indicando los puntos débiles del proceso de enseñanza.

Los autores de la experiencia trabajaron la herramienta teniendo en cuenta las siguientes características: facilidad de uso, adaptabilidad, compatibilidad y robustez que se pueda editar los elementos de evaluación a través de plantillas, recursos para la realización de preguntas, gestión de bases de datos y la posibilidad de usar diversos tipos de cuestionarios. Ellos concluyen que uno de los elementos de autoevaluación para la mejora de la docencia consiste en la realización de un análisis estadístico de los resultados del aprendizaje de los estudiantes.

Hasta ahora la funcionalidad que se ha ido incorporando dentro de los Entornos Integrados de Formación está dirigida a la ayuda al aprendizaje del alumno, bien como complemento a la docencia presencial o bien incluso como plataforma para la educación a distancia. El uso de un entorno de formación vía web constituye un marco ideal para la extracción de información y tratamiento estadístico del proceso de aprendizaje del estudiante. Esto propiciará la aparición de herramientas que utilicen los resultados de la interacción con el alumno para la mejora del proceso de la enseñanza.

El uso de estas nuevas herramientas ligadas al Web abre un nuevo campo en el ámbito educativo, y concretamente en el proceso de autoevaluación de la docencia.

Por último, se encontró otra experiencia por parte de profesores de la Universidad de Oviedo, España, en la cual se usaron herramientas de evaluación online como sistema de aprendizaje para la asignatura de Algoritmia y Lenguajes de Programación de la licenciatura en Matemáticas. Para ello, escogieron dos tipos de herramienta distintos: una que realiza evaluaciones de respuestas múltiples y la herramienta Duck, (una herramienta desarrollada por el Biology Computer Resource Center de la Universidad de Massachusetts Amherst).

Básicamente se trata de un conjunto de scripts PHP3 que permiten implementar un sistema de "cursos". Un curso dispone de una o más áreas incluyendo para cada área varias preguntas y se llevó a cabo un experimento de investigación educativa a fin de determinar, por un lado, las posibilidades de la autoevaluación como sistema de autoaprendizaje y por otro, las posibles ventajas de una herramienta sobre la otra. Duck admite preguntas de respuesta múltiple, 
respuesta corta y de respuesta extensa, estas últimas pueden ser enviadas al tutor para su revisión.

Por otra parte, en Duck no se da ninguna puntuación a las respuestas, puesto que éstas no son ni correctas ni incorrectas; en su lugar, cada respuesta tiene un feedback que sirve para resolver las posibles dudas del estudiante. Por último, en Duck no es necesario seguir una navegación lineal: el usuario puede ir a las preguntas que más le interesen, explorar todas las respuestas, retroceder a preguntas anteriores, etc. Una de las conclusiones que obtuvieron de esta experiencia es que la utilización de herramientas de autoevaluación en el Web facilita a los estudiantes el aprendizaje de conceptos teóricos de asignaturas de programación. La respuesta sobre la preferencia de los estudiantes sobre cuál fue la mejor herramienta quedó pendiente.

Las tres experiencias anteriores tienen en común que sus autores pretenden apoyarse en la autoevaluación para mejorar el proceso de aprendizaje consiguiendo de hecho, buenos resultados con la implementación de éstos recursos. No obstante, se deben hacer más estudios en cuanto a la versatilidad de estas herramientas como recurso de autoevaluación y apoyo para el aprendizaje.

\section{Conclusiones}

La evaluación en la educación es un proceso complejo que abarca desde la práctica institucional hasta la evaluación particular que cada individuo, llámese docente o estudiante, pueda realizar sobre el rol que está desempeñando.

El hecho de que la evaluación sea un proceso complejo implica que los docentes y quienes trabajan en el diseño de material educativo usando herramientas informáticas y tecnología de educación en línea, tengan dificultades para centrar la atención en un tipo específico de evaluación y no se vaya más allá de la evaluación de tipo medición. Es necesario hacer estudios sobre la utilización de la evaluación de tal manera que cumpla la función de ser un recurso que permita mejorar, apoyar y facilitar la construcción de conocimiento.

La autoevaluación aparece de manera general dentro de la teoría que sustenta la Educación a Distancia, pero no se alcanza a percibir realmente la importancia que esta práctica tiene dentro del proceso de enseñanza-aprendizaje -tal vez porque está inmersa dentro de la conceptualización compleja que el término evaluación del aprendizaje conlleva. Esto incide en que, desde el punto de vista teórico no pase de la idea, de que la autoevaluación, es un apoyo que sirve para el estudiante la realice con responsabilidad y autocrítica de manera tal que permita el mejoramiento de aquellos aspectos en los cuales hay debilidades y a su vez determinar las fortalezas que se tiene en cuanto al aprendizaje. En el material 
escrito consultado, se observa la falta de profundidad en este aspecto, pero esto a la vez también proporciona información sobre los aspectos en los cuales se puede profundizar dentro del campo de la Educación a Distancia y de la Tecnología Instruccional.

Existe una gran preocupación creciente por la calidad de los cursos y los instrumentos para evaluar el aprendizaje en cuanto al Diseño Instruccional y las cuestiones tecnológicas. Además se han analizado poco los elementos fundamentales que deben estar presentes en la evaluación del aprendizaje que logran los estudiantes en la Educación a Distancia, a pesar de ser componente esencial constitutivo de dicha modalidad.

Hay necesidad de explorar alternativas en cuanto a software que permita la autoevaluación por parte del estudiante en aras de mejorar el aprendizaje. Hay ejemplos de algunos avances en este sentido pero se debe explorar y realizar más estudios que enriquezcan y fortalezcan la teoría de la evaluación en este sentido.

\section{Referencias bibliográficas}

Barberà, E., (2003). La educación en la red. Actividades virtuales de enseñanza y aprendizaje. Barcelona: Paidós.

Barberà, E. (2006). «Aportaciones de la tecnología a la e-Evaluación». RED. Revista de Educación a Distancia. 5 (6): s.p. Tomado el 28 de marzo de 2010 de http://www. um.es/ead/red/M6

Cabero, J. (2001). La evaluación e investigación sobre los medios de enseñanza. Tecnología Educativa. Diseño y utilización de medios de enseñanza. Barcelona: Paidós.

Cabero, J. y M. Gisbert. (2002). La evaluación. Materiales formativos multimedia en la red. Guía práctica para su diseño. Sevilla: SAV.

Díaz, M.A., et al. (s.f). E-valuate: un modelo de autoevaluación para la mejora de la enseñanza y el aprendizaje. Tomado el 15 de marzo de 2010 de: http://www.di.uniovi. es/ albizu/articulos/jenuio3b.pdf.

Dorrego, E. (2006). «Educación a Distancia y Evaluación del Aprendizaje». RED. Revista de Educación a Distancia. 5 (6): s.p. Tomado el 6 de marzo de 2010 de http:// www.um.es/ead/red/M6

Estévez, C. (1996) Evaluación Integral por Procesos. Santafé de Bogotá: Magisterio. García, A. et al. (2006). «La autoevaluación como actividad docente en entornos 
virtuales de aprendizaje/enseñanza». RED. Revista de Educación a Distancia. 5 (6): s.p. Tomado el 6 de marzo de 2010 de http://www.um.es/ead/red/M6

García Aretio, L. (1998). Indicadores para la evaluación de la enseñanza en una universidad a distancia. Revista Iberoamericana de Educación a Distancia. 1(1): 63-85. García Aretio, L. (2001). La educación a distancia de la teoría a la práctica.Barcelona: Ariel.

García, S. (1996) La evaluación como componente. Santafé de Bogotá: kI PEI Libros y Libros.

Gayo, D. et al. (s.f.) La autoevaluación como método de aprendizaje. Tomado el 2 de marzo de 2010 de http://www.di.uniovi.es/ dani/publications/P012LaGa.PDF

Mantilla, G. (2008). «Diseño y elaboración de módulos autoinstructivos para el Instituto de Educación Superior a Distancia (INSEDI): consideraciones para la evaluación del aprendizaje». Revista Academia y Virtualidad. 1(2): 16-24.

Pérez, M. y G. Bustamante (1996). Evaluación escolar ¿Resultados o procesos? Santafé de Bogotá: Magisterio.

Quesada, R. (2006). «Evaluación del aprendizaje en la educación a distancia "en línea"». RED. Revista de Educación a Distancia. 5 (6) Tomado el 5 de marzo de 2010 de http://www.um.es/ead/red/M6

Rodríguez C., M.J. (2005) «Aplicación de las TIC a la evaluación de alumnos universitarios». Revista Electrónica Teoría de la Educación. 6 (2): s.p. Tomado el día 6 de marzo de 2010 de http://www3.usal.es/ teoriaeducacion

Ryan, S., Scott, B., Freeman, H., y Patel, D. (2000). The Virtual University. London: Kogan Page. 